\title{
5 How do I run STATCALC?
}

There are eight separate programs on the disc. The simplest way to get started is to 'auto-boot' the program called MENU by holding down the SHIFT key and then pressing and releasing the BREAK key. (The !BOOT program is used in this technique, although you do not have to understand anything about it). You will next be presented with a screen of options and, depending upon which option you select, the appropriate program is automatically run. You can then take the disc out of the disc drive and return it to its protective cover.

Alternatively, the programs that MENU selects can be individually CHAINed (or LOADed and RUN) in the usual way. These programs are FILER, COUNTER, UNIVAR, INFER, BIVAR and POLYVAR. Background information and technical advice on these programs is provided in sections 9 to 14 .

\section{What can STATCALC do?}

The options provided by the MENU program are as follows:

(1) Create or alter a data file. Selecting this option will chain the program called FILER.

(2) Analyse frequency data. Selecting this option will chain the program called COUNTER.

(3) Analyse univariate data.

(a) Obtain descriptive statistics or parameters for a single sample or population. Selecting this option will chain the program called UNIVAR.

(b) Obtain significance tests on one or more samples. 